\section{The CD-ROM revolution}

\author{
PETER COOK, Vice-President for Creative \\ Services, Grolier Electronic Publishing Inc., \\ gave the following presentation at the Interna- \\ tional Publishers Association Electronic Pub- \\ lishing Session, Frankfurt, 11 Oct 89.
}

In January 1986 Grolier became the first major publisher to enter the CD-ROM market with its introduction of the Electronic Encyclopedia - a landmark product in the brief history of this exciting new technology. Both we, and the technology, have come a long way since then and in this presentation I will be describing how CD-ROM has evolved over the past 3-4 years and - more importantly - where we think it is going.

\section{Evolution of the technology}

In the early days of CD-ROM the focus was almost entirely on the technology: how many bytes of information a disc could store, the data rate, standards for formatting the data, etc.; and most of the people involved in the business were techies - people from the computer world. I would attend CD-ROM conferences and see only a handful of publishers - the people that own the information that is the ultimate key to the success of CD-ROM.

This is perhaps as it should be, because no new technology emerges mature overnight, and a number of technological issues had to be resolved before the medium could be considered viable. For example, back in 1986 there was no standard for preparing CD-ROM data and no guarantee that different discs could be read on different drives. That problem was resolved by the High Sierra standard and the latest edition of the Grolier encyclopedia - and many other products - can be read on any manufacturer's drive for the IBM PC or the Macintosh.

Another major problem when we published the first edition of the encyclopedia was the lack of retrieval software for accessing the vast amount of information on the disc. Very few companies had software that could match the power of online retrieval services, and publishers had limited alternatives. That issue has also been addressed and at the last count there were over 70 retrieval 'engines' for publishers to choose from and many premastering companies that can take their information and quickly turn it into a CD-ROM.

In the beginning there also wasn't a lot of choice for disc pressing, you either sent your premastered tape to Philips in Eindhoven or Sony in Japan. There are now dozens of facilities that can turn your data into a CD-ROM disc, some even offering a 24-hour turnaround service. In addition, as competition has emerged and the efficiences of the pressing plants have improved - so prices have gone down - discs can now be pressed for under $\$ 2$ each.

In short the technology has matured during the past 4 years and the manufacturing infrastructure is now firmly in place to service the needs of publishers and to build a strong CD-ROM business. However, the technology is not standing still, new capabilities are emerging that could broaden the markets for CD-based products dramatically.

\section{Evolution of the products}

But how are we using the technology? What are publishers placing on CD-ROM to take advantage of the medium? This again is an evolutionary process, driven in part by: experience of the medium itself, economic considerations, and the creative urge to 'stretch' the technology. We view this evolution in 3 waves: -1) Media Conversions. -2) Experimental Mixed Media. -3) Mature Multimedia.

(1) Media conversions. Most CD-ROM products developed during the first wave were direct conversions of online database products, this made economic sense and also recognized that an important early market for CD-ROMs were the same libraries that used online services. Other media conversions included the large libraries of public domain software that were more convenient to distribute on CD-ROM than via floppy discs. The 
media conversion strategy is not without merit because it enabled a number of publishers to get through the initial learning curve and into the business remarkably quickly, without a high financial risk. (Grolier's CD-ROM encyclopedia was converted from an online database file.)

(2) Experimental mixed media. Within a year of those first product releases we were seeing new kinds of CD-ROMs, companies were experimenting with better interfaces, making their products more intuitive and easy to use. Value was also being added to the information by techniques such as 'hypertext', a method of creating cross-references and links within and between databases. And companies began to experiment with graphics and audio, bringing a dimension to $C D-R O M$ products that other distribution media cannot match.

We are still in this experimental phase, learning how to combine databases of text, images, and sound. New tools have been introduced to help us in this evolution: programs such as Apple's HyperCard, which goes a quantum leap beyond the old command line and function key driven software of the first CD-ROM generation. In fact, HyperCard became the vehicle for Grolier's own multimedia experimentation, and was used to produce a prototype for a U.S. History product, which combined text, audio, and image databases from a 50-year period of history.

(3) Mature multimedia. But the third wave of true Multimedia products still lies in the future, because the CD-ROM itself must evolve to standardize the methods for storing images and audio. This will soon be a reality with the emergence next year of CD-ROM XA (extended architecture) which standardizes graphics formats and includes an audio storage method that provides 16 hours of audio on a single $C D$. Other compact disc technologies including CD-I (Compact Disc Interactive) and DVI (Digital Video Interactive) will extend these multimedia capabilities still further.

But CD-ROM is only a part of the multimedia equation, the other key component is the computer required to manipulate the data and, unfortunately, most of the current generation of PCs lack the power and the audiovisual capabilities required for true multimedia. New hardware platforms are evolving, and recent announcements by IBM, MicroSoft, and Intel have outlined the evolutionary phases for multimedia CD-ROM for fu:ure PCs. But the true multimedia computer of the future - the Knowledge Navigator so effectively demonstrated by Apple's John Sculley - remains a distant vision, an inspiration perhaps, but not a viable publishing business for many years to come.

\section{The CD-ROM markets}

To a certain extent, the evolution of the CD-ROM markets has matched that of the technology. As described earlier, most of the initial CD-ROM products in the U.S. and Europe were converted from online databases: medical data, library catalogs, financial data, legal information, etc. - all the popular online categories - quickly migrated to CD-ROM. In the U.S., the library market soon emerged as the largest, for CD-ROM and publishing companies such as Bowker capitalized on this with a range of bibliographic products. In the business market Datext (which eventually became part of Lotus OneSource) became very successful by providing a financial data service, with frequent updates. Some popular reference works, such as Grolier's encyclopedia also became a success, providing schools and libraries with CD-ROM products that children could relate too and use with ease.

Other reference products have since emerged, including the Italian Kronus encyclopedia (which is sold direct to consumers) and the McGraw-Hill Encyclopedia of Science and Technology. Dictionary products include the Oxford English Dictionary, and the Sansyusya multilingual dictionary - which includes 8 languages on the same disc.

But of course it is the business market that must ultimately embrace CD-ROM, and this will gradually happen as PCs proliferate and as CD-ROM is recognized as the most cost effective storage device for all kinds of data. In fact, a number of companies are producing their own CD-ROMs for internal use - particularly for parts catalogs. A recent study of CD-ROM products estimated that more than half the current of CD-ROM titles are in private circulation.

With several hundred CD-ROM titles currently available and with a rapidly growing installed base of CD-ROM drives the indus- 
try is clearly beginning to diversify, presenting publishing opportunities in a number of broad fields as well as niche markets. But the questions that conventional print publishers must ask of themselves before embracing CD-ROM are:

$\mathrm{O}$ Can the technology enhance the value of their information?

$\bigcirc$ Can it help their customers?

$O$ Does it make sound business sense?

When is the right time to make the transition?

Assuming that the first 3 questions can be answered in the affirmative, the last one is always a difficult choice. Print publishers are known for their caution, and most will sit on the fence until CD-ROM becomes the kind of business that can be plotted and predicted with the same degree of certainty as book publishing. But if there is an opening in the market an entrepreneur will emerge to fill it, and not necessarily a publishing company. Many of the successful CD-ROM products are produced by software companies rather than print publishers, and these companies could ultimately become a threat in some fields if publishers are slow to respond to the opportunities of the marketplace.

\section{Future trends}

While the evolution of multimedia will continue to catch the PC press headlines and be the main feature in CD-ROM conferences, it is likely that text applications will provide the vast majority of CD-ROM titles for several years to come. However, even within the constraints of text-only material, the emphasis will be on adding value to both software and the data, increasing the usefulness of the product and enhancing its appeal to the end user.

As the installed base of PCs equipped with CD-ROM drives grows it will become possible to market lower-cost titles that can generate high-volume sales (very few titles have sold in excess of 10,000 units to date). Pergamon Compact Solution have recently announced a multimedia CD-ROM version of the Guinness Book of Records, priced at $\$ 99$. This could well become the first CD-ROM 'hit record', encouraging other publishers to enter the market with well known, attractively priced titles.

The obvious direction for such a trend is for CD-ROM to penetrate the consumer market, but Philips and Sony (the inventors of the compact disc) have already decided that CD-I is the appropriate multimedia technology for consumers because it is designed around a home 'player' rather than a computer. Grolier has elected to develop encyclopedia products around both technologies, believing that $\mathrm{CD}-\mathrm{ROM}$ will dominate in the business market and for schools and libraries while CD-I will eventually emerge as the preeminent consumer CD technology. But whatever the outcome, or the time frame, we as publishers have to look beyond print to take advantage of these emerging technologies and publish our products in the media our customers want. Our encyclopedia is already delivered via online services, videodisc. CD-ROM, and (in 1991) by CD-I, and every single technology has proved commercially viable so far. Furthermore, each has proved enabling for the next technological evolution, and has provided a valuable learning experience that has better equipped our editorial and creative staff to build future generations of electronic encyclopedia products.

Clearly, our experience cannot be viewed as typical, we publish the kind of multivolume reference works that are ideally suited to CD-ROM, but there are significant opportunities for any publisher in the professional reference or popular reference field. In fact, the emergence of multimedia CD-ROM and CD-I should encourage many kinds of publishers: how-to materials, cookery, language learning, travel, educational products, and many more categories, will all become viable if the technology penetrates the consumer market with any degree of success.

In short, we believe that CD-ROM is already a viable medium for a number of publishing categories, even though market growth is likely to be modest for the next 2-3 years. In the long term, the inevitable march of computers into businesses, schools, and homes makes the success of CD-ROM a virtual certainty, and will present an opportunity that publishers should embrace with enthusiasm.

(Reprinted from the IPA Bulletin, No.3, 1989.) 